\title{
For a Cloud computing based Open source E-Health Solution for Emerging Countries
}

\author{
Nabila AISSAOUI \\ Ecole Nationale des Sciences Appliques \\ Mohamed Premier University, \\ OUJDA, MOROCCO.
}

\author{
Mohammed AISSAOUI \\ Ecole Nationale des Sciences Appliques \\ Mohamed Premier University, \\ OUJDA, MOROCCO.
}

\author{
Youssef JABRI \\ Ecole Nationale des Sciences Appliques \\ Mohamed Premier University, \\ OUJDA, MOROCCO.
}

\begin{abstract}
Several studies and statistics have shown that the usage and adoption of E-Health Record software in the emerging countries are very low. This is mainly due to the economic factors, the proprietary E-Health systems are expensive, and requires a longterm maintenance To overcome this budget limitations and to cope with these problems, we propose a method combining the adoption of open-source software, with Cloud computing technology. In this paper we propose a comparative study of the most relevant EHealth open-source software, and select the best suited solution . We propose an implementation of our solution based on openEMR software and the private cloud Openstack.
\end{abstract}

\section{Keywords:}

E -Health, epSOS, DMP, PACS, EHR, EMR, HL7 ,HIPAA, medical information systems, cloud computing, Open source software

\section{INTRODUCTION}

The field of medicine has seen a great evolution in the use of Information and Communication Technologies (ICT). To ensure a best patient care, several applications, such as Picture archiving and communication system (PACS), Electronic Health Record (EHR), Electronic Medical Record (EMR), have emerged and have revolutionized the management of data and services in this area.

Each application is intended to treat one or more parts of the entire health system. And, all of these applications tend to converge and to provide a proper management and treatment of medical data.

Several studies and statistics have shown that the usage and adoption of E-health software are very low. This is mainly due to the economic factor, the proprietary E-health systems are expensive, and require a long-term maintenance [1]. Not to mention the security problem affecting proprietary software, as well as the restriction experienced by the user when trying to switch to another software (data format proprietary software ) [2].

On the other hand, in the most of emerging countries, the annual budget for the use of ICT in health is low and minimal compared to other areas. Furthermore Health institutions are not well equipped with information technology systems. This situation slows the deployment of E-health solutions.

To overcome these budget limitations and to cope with these problems, we propose to combine the adoption of open-source software, with Cloud computing technology.
As an example, taking the study of the Quebec Ministry of Health, which showed that $\$ 18$ million can be stored in 20 years if opensource software are used [3].

Another example, is the implementation, by the health community in western Virginia, of so called the Indian Health System's HER. Known also as Resource and Patient Management System (RPMS) that targets ambulatory care and disease management. The opensource implementation was $51 \%$ cheaper than commercial offers, and maintenance to $66 \%$ cheaper [4].

Actually, there are several open source applications that have been developed, adopted and used by several organisms of health in different countries. We propose in this work to adapt these solutions to the needs of the emerging countries .

As depicted in figure 1 EHR is the heart of health system. Thus we focus our work on this application category.

Nowadays, the cloud computing is admitted as being an important leveraging technology. Consequently, it have to be considered in newest e-health design.

In the rest of this paper, we start by presenting a review of the related work done in this field. After, we discuss the cloud computing concept and its suitability for e-health software purposes. Then, we continue by giving an overview of the most relevant EHR open-source applications, based on their popularity, openness ... And, we present a comparative study of the retained solutions, to select the best suited solution for our needs. We complete consecutively with a discussion of this comparison and experimental results. And we finish with a conclusion and a future work.

\section{RELATED WORKS}

Recently, the Cloud computing paradigm and its use in E-health systems has gained in interest. And several works have addressed the multiple facets of the problems raised in this field.

Firstly, Case studies have been studied in [5], for Health-care information systems (HIS), in Thailand with a transfer of patient information from HCIS to HOSxP (open source HIS) using the standard HL 7. And, in [6] a health care application using Cloud computing and mobile phones is presented. The Interoperability of electronic health records is depicted in [7].

Another Interesting research area, is open-source and E-health. In [2], the authors argue for the superiority of open source licensing to promote safer, more effective health care information systems. 


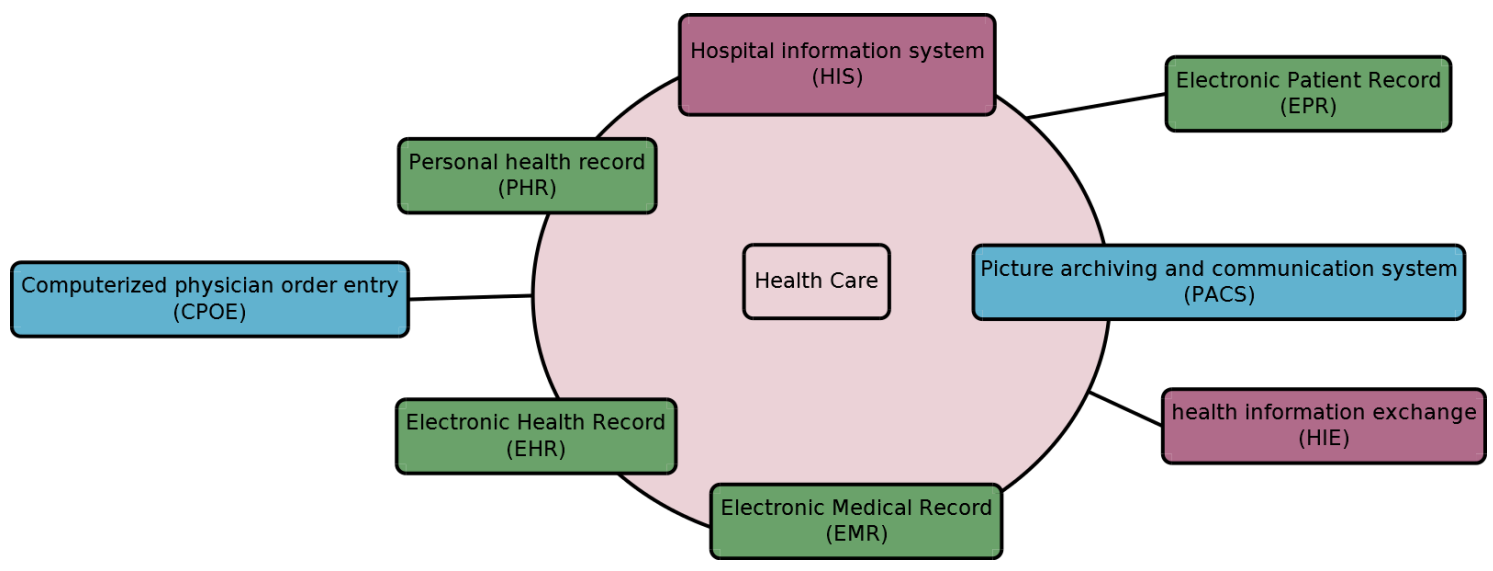

Fig. 1. The E -health applications ecosystem

Authors in [3] discuss the adoption and the use of Open source software in health care organizations.

Secondly, reviews which enriches the experience are presented. A current state and trends of cloud computing in healthcare is explored in [8]. In[9] the concept of e-Health Cloud is introduced. The authors highlight and propose the build of e-health environment and elucidate many of the challenges confronting the success of the e-Health Cloud. The analyze and the examination of cloud computing and its application in the context of optimum health care is provided in [10]. In [11], a cloud-based healthcare system that integrates a formal care system (DACAR) with an informal care system (Microsoft Health Vault) is studied. In [12] the authors present MEDIC, an e-healthcare system which supports both local and centralized access. And in [13] two Cloud-based solutions for different scenarios of Electronic Health Records (EHRs) management system are proposed.

Finally, security and privacy are also relevant to Ehealth system and they have been well addressed. In [14], a review concerning the security and privacy of electronic health record (EHR) systems. In [15], a patient self-controlled access privilege to highly sensitive Personal Health Information is addressed. A patient-centric model of health information exchange is developed in [16]. Privacy issues and storing electronic medical records is discussed in [17].

\section{CLOUD COMPUTING}

Cloud computing is a new concept that brings together all the disciplines, technologies (Web services, virtualization, SOA: service oriented architecture, grid computing,...) and business models used to deliver IT capabilities (software, platforms, hardware) as a service request, scalable and elastic. This is the new trend of computing where IT resources are dynamically scalable, virtualized and exposed as a service on the Internet. [18, 19]. Warranties are offered by the infrastructure provider through tailored service contract: Service Level Agreements (SLA) [18]. Cloud computing consists of three levels of offerings [20, 21]:

(1) Infrastructure as a Service (IaaS), where the equipment is provided in the form of virtual machines. The client maintains the applications, runtimes, integration SOA (Service Oriented Architecture), databases, server software while the supplier maintains the Cloud virtualization, hardware server, storage, networks.
(2) Platform as a Service (PaaS), you can develop your own applications using the services provided. The client maintains only those applications while the supplier maintains the runtimes Cloud, SOA integration, databases, server software, virtualization, server hardware and the storage networks.

(3) Software as a Service (SaaS), the entire applications are available remotely.

The three levels of cloud offering are shown in figure 2, the lower level is the computer hardware resources (computing, storage, network), and mechanisms called virtualization hypervisor, which virtualize access to the material resources of a physical machine (processor, memory and other devices). The interest of a hypervisor is to dynamically add or remove instances of virtual servers on one physical server. This is done using the tools of services and interfaces management. The upper level represents the interactions between the users of the services and the cloud.

There are four different types of deployment of cloud computing [22]:

(1) Public cloud, In the public cloud model, the cloud infrastructure is leased to any class of customers, and the infrastructure is owned by the provider.

(2) Private Cloud, In the model of private cloud infrastructure cloud is operated solely for a single organization with limited access with multiple consumers.

(3) Hybrid Cloud, In the hybrid cloud model, the cloud infrastructure is a composition of two or more forms of clouds (private, community or public) that enable the portability of data and applications.

(4) Community Cloud, in the model of community cloud also called Outsourced private cloud, the cloud infrastructure is provisioned for exclusive use and shared for the specific community (eg the government). It can be owned, managed and operated by one or more organizations in the community.

\section{EHR OPEN SOURCE APPLICATIONS}

There are several open source EHR implementations that are more or less developed than others. Among these, we find SmartCare, ClearHealth, VISTA, vxVISTA and openEMR [23]. These applications not only provide environmental management information data, but also, are based on a sophisticated technology and robust processes. 


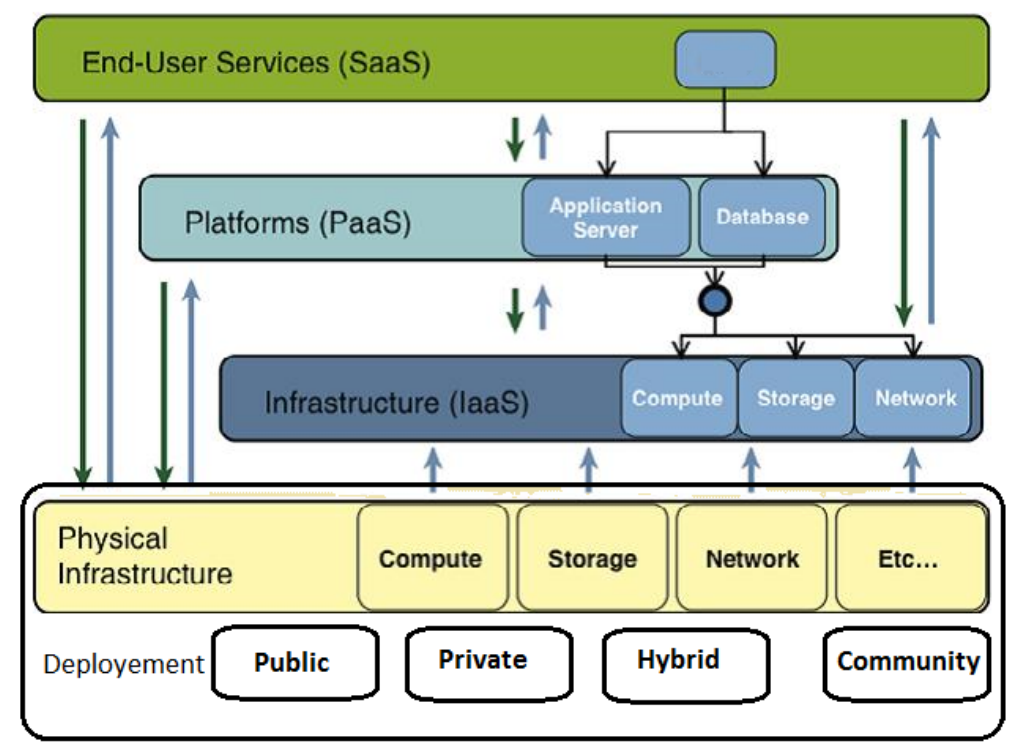

Fig. 2. Cloud computing system

The cost is not the only advantage of these solutions, but also the robustness of their features that is very competitive with proprietary systems.

we select for in our study the last four open source EHR mentioned above; most popular, more sophisticated and offer more features.

\subsection{VISTA ( Veterans Health Information Systems and Technology Architecture )}

VISTA is a robust EHR, developed by the united states department for veterans to support the health system of former military [24] This system manages data from small clinical data to large hospitals. Vista focuses on clinical data archiving, which improves patient care, and enhance clinical and administrative decision making [25].

This system is currently used by hundreds of former military clinics in the U.S. and also has been adopted by the Department of Defense in the same country, by the Department of Health and Human Service India, by Mexico Institute - Instituto Mexicano del Seguro Social and the National cancer Institute at the University of Cairo, Egypt.

VISTA is developed with the MUMPS language which is specifically designed for medical systems [26]. This language suffers from limitations, especially in the graphical interfaces, but it can communicate with other languages that guarantee that VISTA is supported online by a Vistapedia and a wiki system.

\section{2 vxVISTA}

vxVista is an improved version of Vista by DSS Inc, to deliver additional functions, not only VA release. Several modules have been added in this version of vista, among these [27], we find:

-Administration of medication codes (VxBCMA).

-Pharmacy for in-patient and out-patient.

An in-patient, is a patient who is admitted to hospital and stayed for several days. His care is called inpatient care. The out-patient is a patient who is not hospitalized but just visit the organization of health for treatment or diagnosis and does not necessarily demand the administrative procedures, the type of care received by the patient. The out-patient is also known as ambulatory care. vxVista was certified in-patient use. vx Vista architecture is a patient's centric and it is developed under the Eclipse Public License. vxVista is developed using the same MUMPS language, such VISTA. And it inherit the same weaknesses.

\subsection{ClearHealth}

ClearHealth is one of the most popular open source EHR, and is used in hundreds of hospitals worldwide. Clearhealth supports several features including, planning, support for recording patient demographic, electronic billing, EMR, and a reporting system based on SQL.

ClearHealth has been identified as a viable solution for the foundation of health care in California (the california healthcare foundation) [28]. This EHR uses, HL7 protocol that ensures interoperability of the system, and HIPAA protocol for security. ClearHealth is developed in the PHP language.

\subsection{OpenEMR}

OpenEMR is a multi-platform open source HER and electronic management record (EMR) application.

OpenEMR is intended for outpatient and ambulatory practices and is certified ONC full. The system is operable in different platform. OpenEMR connectivity and interoperability is supported with HL7 protocol Interface [29].

The software interface is simple and user-friendly and offers several features [30], such as management of patient data ,schedule of visits, EMR, billing management and report Management. For interoperability, OpenEMR is also a HIPAA compliant [31].

\section{COMPARATIVE STUDY OF EHR SOLUTIONS}

In order to select the most appropriate solution to our needs, we propose a comparative study of the applications presented in section 4 . We define some comparative criteria for this study. 
Table 1. Comparative study of EHR solutions.

\begin{tabular}{lllll}
\hline \hline & VISTA & vxVista & OpenEMR & Clear health \\
\hline Open-source & $*$ & $*$ & $*$ & $*$ \\
Modularity & $*$ & $*$ & $*$ & $*$ \\
Interoperability & $*$ & $*$ & $*$ & $*$ \\
support & Vistapedia & FAQ, Doc, web, FAQ, Doc, web, very large FAQ, Doc, web, large & Fommunity \\
& & community & community & Eclipse Public \\
License & GPL & Eclipse Public & Eclipse Public & PHP \\
Language & MUMPS & MUMPS & PHP & All \\
Platform & Native & Unix-like Windows & All & Web-based \\
Client type & Native & Native & Web-based & \\
\hline
\end{tabular}

The most important criteria, are popularity, community use, modularity, openness, security and support.

In addition to that, a free open-source software, an independence from the software provider and a wide possibility of setting the applications according to the needs of users are also a crucial requirements.

\subsection{Modularity}

The modular architecture, is an architecture where at least some components are optional, and have the ability to add or remove modules according to the needs.

A modular application is therefore based on this principle and gives the user the ability to create different configuration by suppressing or adding modules (add-on).

These modules can be developed, tested and maintained separately and added without reinstalling the software. In this way, the users can implement the module they need without having to acquire the entire system.

Modularity is a necessary feature in a health system.

\subsection{Interoperability}

The interoperability is the ability of a system to communicate with another system without restriction. It aims to be compatible with different systems by defining rules for operations and for sharing data. The Interoperability aim that the system could be operated in different environments, and also accessible from different locations.

In order assure the interoperability of the system, the applications have to comply with standards such HL7 [32]. We note also that, epSOS (Smart Open Services for European Patients) [33] is another interoperability project. Its goal is to enable European citizens to access their health information outside their country. We conclude that the interoperability feature is crucial in the field of E-health applications.

\subsection{Security}

The data security issues arise in all areas, even in the field of health. The processed data are, private patient data, their health status, their personal details, and the various interventions experienced. Computer applications related to health have to provide a high level of security, in order to ensure the confidentiality and the integrity of data.

Indeed, the security dilemma is one of the main causes of the minimal rate of cloud adoption by healthcare professionals . Professionals demand that cloud providers meet and safety standards to ensure the desired level of security. Among these we find the HIPAA standard (Health Insurance Portability and Accountability Act ) [34].

\subsection{Discussion}

In the table 11, we summarize the results obtained from our comparative study.

Several considerations should be taken to determine the best suited solution for the implementation of an EHR E-Health system.

Firstly, we consider the openness aspect. All the application presented in this study are fully open source, and their source code are freely available.

Modularity and interoperability are also satisfied by the applications. They also complain with modularity standards.

OpenEMR and Clearhealth are developed in PHP. That guarantee compatibility with web standards. We consider this as a strong feature, in contrast with MUMPS which is a specific language with intrinsic limitations.

In addition to that, OpenEMR and Clearhealth are multiplatform and directly used with a web client. Vista and vxVista necessitate a specific client and are accessible only from windows and unix like platfroms. This limits the use from practically all the handled and mobile devices.

Both of openEMR and Clearhealth present interesting features. Our final selection is based on community use. That is very important criterion since the application could evolve and react rapidly to different issues.

We find that OpenEMR community is very active and large. The online help is very simple and intuitive.

According to this discussion, OpenEMR seems to be the solution that satisfy our initial requirements, and best meets the demands of healthcare organizations.

With all these features, OpenEMR is well suited to a future deployment to the cloud.

\section{SOLUTION IMPLEMENTATION}

In this section, we present the feasibility study of the cloud based OpenEMR solution. We started by setting up a testbed for hosting the E-health application.

We opted for the OpentStack solution as a cloud platform [35]. OpenStack is recent and under active development. It has great potential due to its architecture and large community and the support of its partners. All code is licensed under Apache 2 license. Openstack is supported by many companies in the world and is based on the code used by NASA and Rackspace Cloud. It is written in python and currently implements two control APIs, the EC2 API and Rackspace. It uses different drivers to interface with a maximum number of hypervisors (Xen, KVM, HyperV, Qemu) [36]. This project is dedicated to providing the computer 


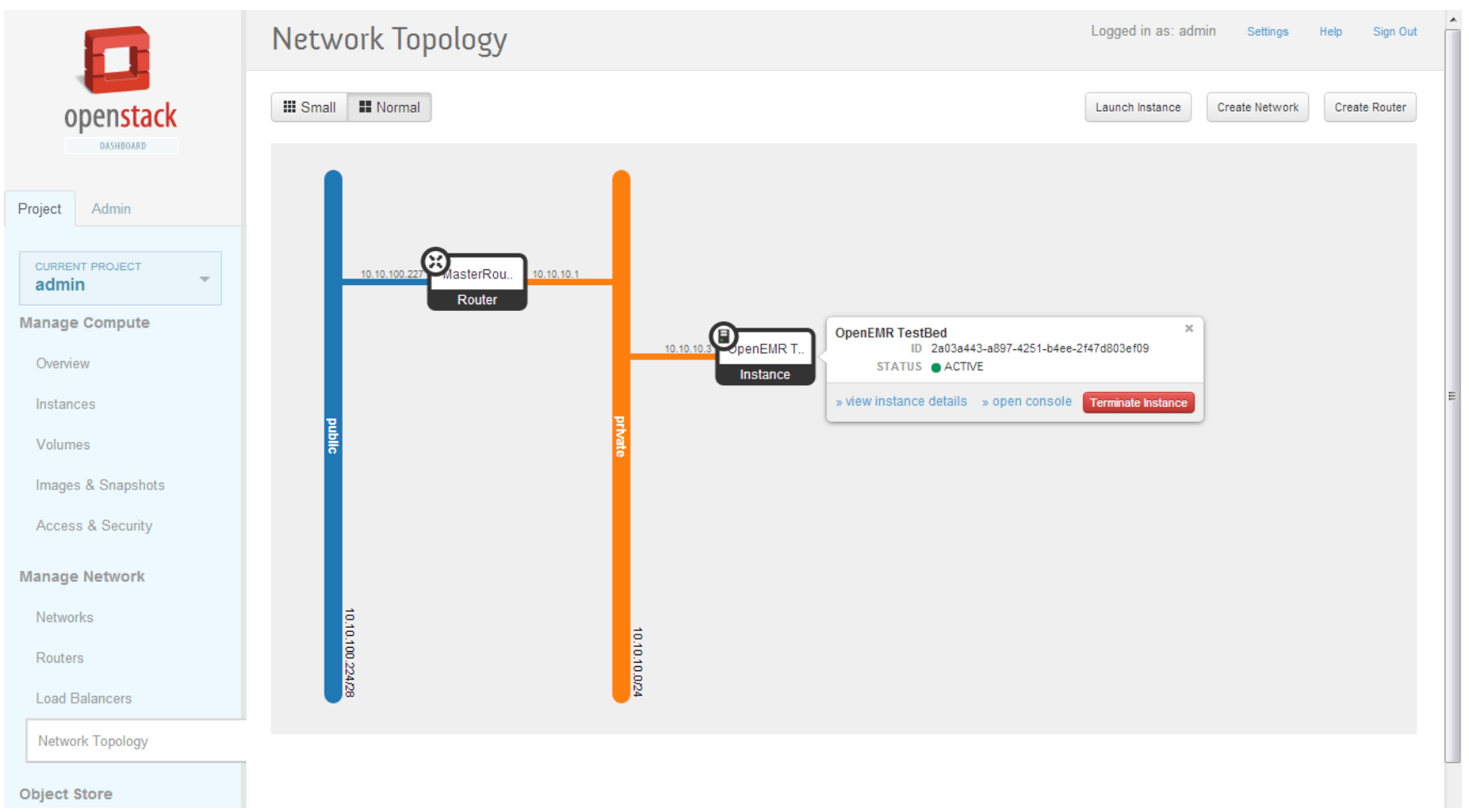

Fig. 3. OpenERM testbed solution

industry with the opportunity to build a hosting architecture and massive scalability and is completely open source, while it overcomes the constraints of the use of proprietary technologies. Currently, we have deployed the OpenEMR application as a virtual instance running on the openstack testbed platform(figure 3). The application is directly accessible to the users through a simple web navigator. This system will be used to evaluate the application performances.

\section{CONCLUSION}

In this paper, we discussed our contribution to the effort of the development of cloud e-health solutions for emerging countries. We proposed to combine the adoption of open-source software, with Cloud computing technology.

We also conducted a comparative study of the most used opensource ehealth applications to select best suited to Cloud adoption.

We demonstrate the feasibility of our approach by deploying the retained solution on the openstack testbed platform.

We conclude that the Cloud technology combined with opensource software, appears to be an effective initiator for the adoption of the E-health applications.

As future work, we intend to continue with a consolidation work in order to add missing features. And finally to migrate the overall system to PaaS based architecture platform

\section{REFERENCES}

[1] Samuel Bowen MD, Robert Hoyt MD, Ladeana Glenn RN, BSN Donald McCormick, and Xavier Gonzalez. Open-source electronic health records: Practice implications, February 2011. http://www.oemr.org/wiki/Open_Source_EHR_in_Practice.

[2] J. Carl Reynolds and C. Jeremy Wyatt. Open source, open standards, and health care information systems. J Med Internet Res, 13(1):e24, Feb 2011.

[3] Josianne Marsan and Guy Par. Antecedents of open source software adoption in health care organizations: A qualitative survey of experts in Canada. 82:731-741.

[4] Robert H Miller and Ida Sim. Physicians use of electronic medical records: barriers and solutions. Health affairs, 23(2):116-126, 2004.

[5] S. Ngamsuriyaroj, C. Sirichamchaikul, S. Hanam, and T. Tatsanaboonya. Patient information exchange via web services in hl $7 \mathrm{v} 3$ for two different healthcare systems. In Computer Science and Software Engineering (JCSSE), 2011 Eighth International Joint Conference on, pages 420-425, 2011.

[6] M.D. Lakshmi and J.P.M. Dhas. An open source private cloud solution for rural healthcare. In Signal Processing, Communication, Computing and Networking Technologies (ICSCCN), 2011 International Conference on, pages 670674,2011

[7] S. Garde, P. Knaup, E. J. S. Hovenga, and S. Heard. Towards semantic interoperability for electronic health records. 46:332-343.

[8] Sanjay P Ahuja, Sindhu Mani, and Jesus Zambrano. A survey of the state of cloud computing in healthcare. Network and Communication Technologies, 1(2):p12, 2012. 
[9] Eman AbuKhousa, Nader Mohamed, and Jameela Al-Jaroodi. e-health cloud: Opportunities and challenges. Future Internet, 4(3):621-645, 2012.

[10] S.K. Chowdhary, A. Yadav, and N. Garg. Cloud computing: Future prospect for e-health. In Electronics Computer Technology (ICECT), 2011 3rd International Conference on, volume 3, pages 297-299, 2011.

[11] E. Ekonomou, L. Fan, W. Buchanan, and C. Thuemmler. An integrated cloud-based healthcare infrastructure. In Cloud Computing Technology and Science (CloudCom), 2011 IEEE Third International Conference on, pages 532-536, 2011.

[12] D. Patra, S. Ray, J. Mukhopadhyay, B. Majumdar, and A.K. Majumdar. Achieving e-health care in a distributed ehr system. In e-Health Networking, Applications and Services, 2009. Healthcom 2009. 11th International Conference on, pages 101-107, 2009.

[13] Gonzalo Fernández-Cardeñosa, Isabel de la Torre-Díez, Miguel López-Coronado, and Joel JPC Rodrigues. Analysis of cloud-based solutions on ehrs systems in different scenarios. Journal of medical systems, 36(6):3777-3782, 2012.

[14] Jos Luis Fernndez-Alemn, Inmaculada Carrin Seor, Pedro ngel Oliver Lozoya, and Ambrosio Toval. Security and privacy in electronic health records: A systematic literature review. Journal of Biomedical Informatics, 46(3):541 - 562, 2013.

[15] Mrinmoy Barua, Xiaohui Liang, Rongxing Lu, and Xuemin Shen. ESPAC: enabling security and patient-centric access control for eHealth in cloud computing. International Journal of Security and Networks, 6(2):67-76, January 2011.

[16] Ming Li, Shucheng Yu, Yao Zheng, Kui Ren, and Wenjing Lou. Scalable and secure sharing of personal health records in cloud computing using attribute-based encryption. Parallel and Distributed Systems, IEEE Transactions on, 24(1):131143,2013

[17] Zhuo-Rong Li, En-Chi Chang, Kuo-Hsuan Huang, and Feipei Lai. A secure electronic medical record sharing mechanism in the cloud computing platform. In Consumer Electronics (ISCE), 2011 IEEE 15th International Symposium on, pages 98-103, 2011.

[18] Lutz Schubert, Keith G Jeffery, and Burkard NeideckerLutz. The Future of Cloud Computing: Opportunities for European Cloud Computing Beyond 2010:-expert Group Report. European Commission, Information Society and Media, 2010.

[19] Lung-Hsing Kuo, Jui-Chen Yu, Hsieh-Hua Yang, Wen-Chen $\mathrm{Hu}$, and Hung-Jen Yang. A study of creating technology education course for cloud computing. INTERNATIONAL JOURNAL OF Communications, 6:11, 2012.

[20] Marc Jansen. Will cloud computing change standards in it-service management? Journal of Communication and Computer, 9(7):813-823, 2012.

[21] Paul Pocatilu, Felician Alecu, and Marius Vetrici. Measuring the efficiency of cloud computing for e-learning systems. WSEAS Transactions on Computers, 9(1):42-51, 2010.

[22] Qi Zhang, Lu Cheng, and Raouf Boutaba. Cloud computing: state-of-the-art and research challenges. Journal of Internet Services and Applications, 1(1):7-18, 2010.
[23] Top open source medical billing and emr software, June 2012. http://linuxaria.com/recensioni/top-open-sourcemedical-billing-and-emr-software?lang=en.

[24] James Herbsleb, Claudia MüllerBirn, and W Ben Towne The vista ecosystem: Current status and future directions. Pittsburgh, PA: Institute for Software Research, 2010.

[25] Alan Naditz. Telemedicine at the va: Vista, myhealthevet, and other va programs. Telemedicine and e-Health, 14(4):330332,2008

[26] GO Barnett, DE Souder, J Bowie, and Norma Justice. Mumps: A support for medical information systems. Informatics for Health and Social Care, 1(3):183-189, 1976.

[27] vxvista. http://www.openhealthnews.com/content/vxvista.

[28] Clearhealth. http://en.wikipedia.org/wiki/ClearHealth.

[29] Openemr connectivity and interoperability, 20122012 http://www.open-emr.org/wiki/index.php/Visolve.

[30] Dan ISTRATE and Dumitru MOLDOVAN. The free software model of development in the area of medical informatics. Applied Medical Informatics, 31(4), 2013.

[31] DART Notatnr, Forfattere Wolfgang Leister, and Per Røe. A short memo on open source software for pacs.

[32] Robert H Dolin, Liora Alschuler, Sandy Boyer, Calvin Beebe, Fred M Behlen, Paul V Biron, and Amnon Shabo Shvo. H17 clinical document architecture, release 2. Journal of the American Medical Informatics Association, 13(1):30-39, 2006.

[33] Fredrik Linden. epsos local data providers. Acta Informatica Medica, 17(3):142-145, 2009.

[34] George J Annas. Hipaa regulations-a new era of medicalrecord privacy? New England Journal of Medicine, 348(15):1486-1490, 2003.

[35] Omar Sefraoui, Mohammed Aissaoui, and Mohsine Eleuldj. Openstack: Toward an open-source solution for cloud computing. International Journal of Computer Applications, 55(3):38-42, October 2012. Published by Foundation of Computer Science, New York, USA.

[36] Omar Sefraoui, Mohammed Aissaoui, and Mohsine Eleuldj. Comparison of multiple iaas cloud platform solutions. Proceedings of the 7th WSEAS International Conference on Computer Engineering and Applications, (Milan-CEA '13), ISBN: 978-1-61804-150-0, 2013. 